\title{
Quantum mechanics needs no interpretation
}

\author{
L. Skála ${ }^{1,2 *}$ and V. Kapsa ${ }^{1}$ \\ ${ }^{1}$ Charles University, Faculty of Mathematics and Physics, \\ Ke Karlovu 3, 12116 Prague 2, Czech Republic and \\ ${ }^{2}$ University of Waterloo, Department of Applied Mathematics, \\ Waterloo, Ontario N2L 3G1, Canada
}

\begin{abstract}
Probabilistic description of results of measurements and its consequences for understanding quantum mechanics are discussed. It is shown that the basic mathematical structure of quantum mechanics like the probability amplitude, Born rule, probability density current, commutation relations, momentum operator, uncertainty relations, rules for including the scalar and vector potentials and existence of antiparticles can be derived from the definition of the mean values of the space coordinates and time. Equations of motion of quantum mechanics, the Klein-Gordon equation, Schrödinger equation and Dirac equation are obtained from requirement of the relativistic invariance of the theory. Limit case of localized probability densities leads to the Hamilton-Jacobi equation of classical mechanics. Many particle systems are also discussed.
\end{abstract}

keywords: probability theory, quantum mechanics, classical mechanics PACS 03.65-w, 03.65.Ca, 03.65.Ta

\section{INTRODUCTION}

Quantum mechanics is one of the most thoroughly tested physical theories (see e.g. [1, 2, 3]). At the same time, standard approach to introducing quantum mechanics based on the sometimes contra-intuitive postulates has rather mathematical than physical character and exact physical meaning of the postulates and their interpretation is subject of continuing discussion. In this approach, quantum mechanics appears as a field with strange paradoxes and phenomena that are not easy to understand (see e.g. [4]). It is not satisfactory and, in our opinion, it is time (after almost 80 years after formulating the basic principles of quantum mechanics) to replace this approach by a more physical one based on a more direct description of measurements. Only such approach can clarify physical meaning of numerous (sometimes hidden) assumptions made in quantum mechanics. Paraphrasing the title of the recent paper by Fuchs and Peres [5] we can say: Quantum theory needs no 'interpretation' - it needs derivation from description of measurements. In this sense, our approach can be understood as extension and justification of the standard interpretation of quantum mechanics.

We note that our approach is different from that used usually in physics: To explain experimental results, one introduces some physical quantities and evolution equations these quantities have to obey. Then, consequences of these equations are investigated and compared with experiment. In our approach, we describe results of measurements in a probabilistic way and ask what is the mathematical apparatus that can describe this situation. In this way, the basic mathematical structure of quantum theory except for equations of motion is obtained. Equations of motion are found from requirement of the relativistic invariance of the probabilistic description.

Probably the best approach is to start with measurement of the space coordinates and time. In this paper, we show that the basic mathematical structure of quantum mechanics like the probability amplitude, Born rule, probability density current, commutation relations, momentum operator, uncertainty relations, rules for including the scalar and vector potentials and existence of antiparticles can be derived from the definition of the mean values of the space coordinates and time (sections 【II). Equations of motion of quantum mechanics, the Klein-Gordon equation, Schrödinger equation and Dirac equation are obtained from requirement of the relativistic invariance of the theory (section VIII). Limit case of localized probability densities yields the Hamilton-Jacobi equation of classical mechanics (section VIII). Generalization to many particle systems is performed in section IX

\section{UNCERTAINTY RELATION FOR PROBABILITY DENSITY}

Physical measurements are imperfect and repeated measurements of the same quantity under the same experimental conditions yield different results. The most simple characteristics of such measurements is the mean value of the results

\footnotetext{
* Corresponding author. E-mail: skala@karlov.mff.cuni.cz
} 
of repeated measurements. It is the starting point of the following discussion.

We assume that the mean value $\langle x\rangle$ of the measurement of the coordinate $x$ is given by the usual definition

$$
\langle x\rangle=\int x \rho(\mathbf{r}) \mathrm{d} V,
$$

where the integration is carried out over the whole space and $\rho(\mathbf{r}) \geq 0$ is the normalized probability density

$$
\int \rho \mathrm{d} V=1
$$

First, we use integration by parts with respect to the variable $x$ in Eq. (2) and get

$$
\left.x \rho\right|_{x=-\infty} ^{\infty}-\int x \frac{\partial \rho}{\partial x} \mathrm{~d} V=1 .
$$

Assuming that the first term in this equation equals zero for physically reasonable $\rho$ we obtain the starting point of the following discussion

$$
\int x \frac{\partial \rho}{\partial x} \mathrm{~d} V=-1
$$

The last equation can be rewritten in form of the inner product

$$
(u, v)=-1
$$

defined in the usual way

$$
(u, v)=\int u^{*}(\mathbf{r}) v(\mathbf{r}) \mathrm{d} V
$$

Here, the star denotes the complex conjugate and functions $u$ and $v$ equal

$$
\begin{gathered}
u=x \sqrt{\rho}, \\
v=\frac{1}{\sqrt{\rho}} \frac{\partial \rho}{\partial x},
\end{gathered}
$$

where $s=s(\mathbf{r})$ is a real function. Using the Schwarz inequality

$$
(u, u)(v, v) \geq|(u, v)|^{2}
$$

and Eqs. (5)-(8) we can derive the uncertainty relation in the form

$$
\int x^{2} \rho \mathrm{d} V \int \frac{1}{\rho}\left(\frac{\partial \rho}{\partial x}\right)^{2} \mathrm{~d} V \geq 1,
$$

where the second integral is the so-called Fisher information [6, 7, 8, 9]. By using Eq. (4) and the condition $\rho \rightarrow 0$ for $x \rightarrow \pm \infty$ this result can be generalized to the Cramer-Rao inequality [8, 10]

$$
\int(x-\langle x\rangle)^{2} \rho \mathrm{d} V \int \frac{1}{\rho}\left(\frac{\partial \rho}{\partial x}\right)^{2} \mathrm{~d} V \geq 1 .
$$

In agreement with standard statistical description of results of measurements, we require here the same weight function $\rho$ in the integrals $\int x \rho \mathrm{d} V, \int x^{2} \rho \mathrm{d} V$ and $\int(x-\langle x\rangle)^{2} \rho \mathrm{d} V$. Assumptions (7) and (8) obey this requirement.

Uncertainty relation (11) is general consequence of Eq. (1) and shows that uncertainty relations must appear in any probabilistic theory of this kind, including quantum mechanics. There are two important quantities appearing in the uncertainty relation (11): the coordinate $x$ and the derivative $\partial / \partial x$. Similar quantities, namely the coordinate $x$

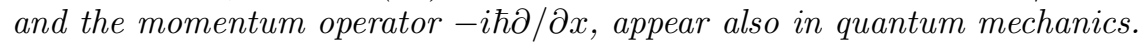




\section{BORN RULE AND COMPLEX PROBABILITY AMPLITUDE}

In the preceding section, we made use of the expression $\rho=(\sqrt{\rho})^{2}$ in the inner product $(u, u)$ and derived the uncertainty relations (10) and (11). However, we can assume also a general expression

$$
\rho=\psi^{*} \psi
$$

where $\psi$ is a complex probability amplitude with the property

$$
|\psi|=\sqrt{\rho}
$$

Equation (12) agrees with the well-known Born rule for calculating the probability density from the probability amplitude.

Physical meaning of the complex form of $\psi$ can be elucidated as follows. To describe physical systems, we have to specify not only the space-time probability distribution $\rho$, but also its evolution in space-time. This information can be encoded into the complex part of the probability amplitude $\psi$. Here, we can proceed similarly as in continuum mechanics, where not only the density $\rho$ but also the density current

$$
j_{k}=\rho v_{k}, \quad k=1,2,3
$$

related to the components of the velocity $v_{k}$ is introduced. Writing the "velocity" in the form

$$
v_{k}=\frac{\partial s}{\partial x^{k}}
$$

where $s=s(\mathbf{r}, t)$ is a real function we get

$$
j_{k}=\rho \frac{\partial s}{\partial x^{k}}=\sqrt{\rho} \mathrm{e}^{-i s} \sqrt{\rho} \mathrm{e}^{i s} \frac{\partial s}{\partial x^{k}}=\sqrt{\rho} \mathrm{e}^{-i s}(-i) \frac{\partial\left(\sqrt{\rho} \mathrm{e}^{i s}\right)}{\partial x^{k}}+\frac{i}{2} \frac{\partial \rho}{\partial x^{k}} .
$$

Now, introducing the complex probability amplitude in agreement with Eqs. (12)-(13)

$$
\psi(\mathbf{r}, t)=\sqrt{\rho(\mathbf{r}, t)} \mathrm{e}^{i s(\mathbf{r}, t)}
$$

we have

$$
j_{k}=\psi^{*}\left(-i \frac{\partial \psi}{\partial x^{k}}\right)+\frac{i}{2} \frac{\partial \rho}{\partial x^{k}} .
$$

However, the probability density current has to be real. Calculating the real part of $j_{k}$ we obtain the final expression

$$
j_{k}=\frac{1}{2}\left[\psi^{*}\left(-i \frac{\partial \psi}{\partial x^{k}}\right)+c . c .\right]=\frac{1}{2 i}\left(\psi^{*} \frac{\partial \psi}{\partial x^{k}}-\psi \frac{\partial \psi^{*}}{\partial x^{k}}\right) .
$$

Except for a multiplicative factor, this formula agrees with the expression for the probability density current known from quantum mechanics. The complex probability amplitudes $\psi$ are necessary to obtain nonzero $j_{k}$. The probability density current depends on the operator $-i \partial / \partial x^{k}$. Except for the factor $\hbar$, this operator agrees with the momentum operator $-i \hbar \partial / \partial x^{k}$ known from quantum mechanics. Therefore, this part of quantum mechanics can be derived from general properties of the probability theory. In agreement with rules of quantum mechanics, the probability amplitudes $\psi$ and $\psi \exp (i \alpha)$, where $\alpha$ is a real constant, yield the same probability density $\rho$ and probability density current $j_{k}$.

\section{COMMUTATION RELATIONS FOR THE COORDINATE AND MOMENTUM}

Now we use Eqs. (4) and (12) and get

$$
\int x\left(\frac{\partial \psi^{*}}{\partial x} \psi+\psi^{*} \frac{\partial \psi}{\partial x}\right) \mathrm{d} V=-1
$$

Multiplying this equation by $-i$ we obtain the equation

$$
\int\left[(x \psi)^{*}\left(-i \frac{\partial \psi}{\partial x}\right)-\left(-i \frac{\partial \psi}{\partial x}\right)^{*} x \psi\right] \mathrm{d} V=i
$$

or

$$
[x,-i \partial / \partial x]=i .
$$

Except for the factor $\hbar \neq 0$, this commutation relation agrees with the commutation relation between the coordinate and momentum known from quantum mechanics. 


\section{UNCERTAINTY RELATIONS FOR THE COORDINATE AND MOMENTUM}

Equation (20) can be rewritten as

$$
2 \operatorname{Re}(u, v)=-1
$$

where $u=x \psi$ and $v=\partial \psi / \partial x$. Calculating the square of Eq. (23) we get successively

$$
\begin{gathered}
1=4[\operatorname{Re}(u, v)]^{2} \leq \\
\leq 4\left\{[\operatorname{Re}(u, v)]^{2}+[\operatorname{Im}(u, v)]^{2}\right\}=4|(u, v)|^{2} .
\end{gathered}
$$

From this result and the Schwarz inequality (9) we obtain the relation

$$
(u, u)(v, v) \geq \frac{1}{4}
$$

that can be written in form of the uncertainty relation

$$
\int x^{2}|\psi|^{2} \mathrm{~d} V \int|-i \partial \psi / \partial x|^{2} \mathrm{~d} V \geq \frac{1}{4} .
$$

This result can be further generalized. Using the integration by parts and the condition $\rho \rightarrow 0$ for $x \rightarrow \pm \infty$ Eq. (20) can be generalized as

$$
\begin{gathered}
\int[(x-a) \psi]^{*}\left[\frac{\partial \psi}{\partial x}-i b \psi\right] \mathrm{d} V+ \\
+\int\left[\frac{\partial \psi}{\partial x}-i b \psi\right]^{*}[(x-a) \psi] \mathrm{d} V=-1,
\end{gathered}
$$

where $a$ and $b$ are real constants. From this equation, a more general form of the uncertainty relation can be obtained

$$
\int(x-a)^{2}|\psi|^{2} \mathrm{~d} V \int|-i \partial \psi / \partial x-b \psi|^{2} \mathrm{~d} V \geq \frac{1}{4} .
$$

The minimum of the left side is obtained for

$$
a=\int \psi^{*} x \psi \mathrm{d} V=\langle x\rangle
$$

and

$$
b=\int \psi^{*}(-i \partial \psi / \partial x) \mathrm{d} V=\langle-i \partial / \partial x\rangle .
$$

Except for the factor $\hbar$, the resulting uncertainty relation with a and $b$ given by the last two equations agrees with the well-known Heisenberg uncertainty relation. Again, it follows from Eqs. (4) and (12).

It is worth noting that Eq. (27) remains valid also in case if $b$ is a real function $b=f_{x}(\mathbf{r}, t)$. It means that the operator $-i \partial \psi / \partial x$ can be replaced by the operator $-i \partial \psi / \partial x-f_{x}$ and the commutation relation (22) and the uncertainty relation (28) can be further generalized. Therefore, general structure of the probability theory remains preserved for any real function $f_{x}$. In physics, different functions $f_{x}, f_{y}$ and $f_{z}$ correspond to different components of the electromagnetic vector potential $\mathbf{A}=\left(A_{x}, A_{y}, A_{z}\right)$ multiplied by the charge $e$ of the particle. Except for $\hbar$ and $e$, we obtained the rule $-i \hbar \nabla \rightarrow-i \hbar \nabla-e \mathbf{A}$ for including the vector potential $\mathbf{A}$ into quantum theory (charge is discussed at the end of section (VI). We note also that the kinetic energy in quantum mechanics $T=\left(\hbar^{2} / 2 m\right) \int|\nabla \psi|^{2} \mathrm{~d} V$ equals the space Fisher information $\int|\nabla \rho|^{2} / \rho \mathrm{d} V$ multiplied by $\hbar^{2} /(8 m)$. 


\section{TIME}

Time can be discussed similarly as the space coordinates, however, there are some important differences that has to be taken into consideration.

Assuming that there are given initial conditions for $\psi(\mathbf{r}, t=0)$ the probability amplitude $\psi(\mathbf{r}, t), t>0$ gives the probability description of measurements at later times. Therefore, time evolution has unidirectional character from given initial conditions to the relative probability of results of (yet unperformed) measurements at later times. If this measurement is actually performed, the probabilistic description must be replaced by a concrete result following from the performed measurement. It is the basis of two different evolution schemes in quantum mechanics: time evolution described by the evolution equation like the Schrödinger equation and reduction or collapse of the wave function. In this paper, we are interested in the former case. Detailed description of the reduction of the probability amplitude is not needed in our approach.

In standard quantum mechanics, the probability amplitudes obey the normalization condition $\int|\psi|^{2} \mathrm{~d} V=1$ valid at all times and the integral over time $\int_{0}^{\infty} \int|\psi|^{2} \mathrm{~d} V \mathrm{~d} t$ goes to infinity. This situation can be compared to that for a free particle. For a free particle, the integral $\int|\psi|^{2} \mathrm{~d} V$ goes to infinity and $\psi$ is usually normalized by means of the Dirac $\delta$-function. For time, similar approach cannot be used for two reasons. First, we do not perform here integration over all times, but from the initial condition at $t=0$ to infinity. Second, if the integral $\int_{0}^{\infty} \int|\psi|^{2} \mathrm{~d} V \mathrm{~d} t$ goes to infinity we cannot define the mean time by analogy with Eq. (11) and proceed similarly as in the preceding sections. For these reasons, we assume that not only the integral $\int|\psi|^{2} \mathrm{~d} V$ but also the integral

$$
\int_{0}^{\infty} \int|\psi|^{2} \mathrm{~d} V \mathrm{~d} t=1
$$

equals one and proceed by analogy with the space coordinates. In this way, we get the operator $i \partial / \partial t$, obtain the corresponding commutation and uncertainty relations and introduce the scalar potential. At the end of our discussion, we will assume that $\int|\psi|^{2} \mathrm{~d} V$ changes negligibly in time, normalize the probability amplitude by means of the usual condition $\int|\psi|^{2} \mathrm{~d} V=1$ and perform transition to standard quantum mechanics.

First, we define the time component of the probability density current by the equation analogous to Eqs. (14)-(15)

$$
j_{t}=-\rho \frac{\partial s}{\partial t}
$$

and obtain expression similar to Eq. (19)

$$
j_{t}=\frac{1}{2}\left[\psi^{*}\left(i \frac{\partial \psi}{\partial t}\right)+\text { c.c. }\right]
$$

Except for a factor, this quantity equals the time component of the probability density current $j_{0}=$ $\operatorname{Re}\left[\psi^{*} i \hbar\left(\partial \psi / \partial x^{0}\right)\right] / m_{0}$ known from relativistic quantum mechanics, where $x^{0}=c t$ and $m_{0}$ is the rest mass. Then, by analogy with Eq. (21) we derive the equation

$$
\int_{t=0}^{\infty} \int\left[\left(i \frac{\partial \psi}{\partial t}\right)^{*} t \psi-(t \psi)^{*}\left(i \frac{\partial \psi}{\partial t}\right)\right] \mathrm{d} V \mathrm{~d} t=i .
$$

One can introduce also a real constant $d$ into this equation

$$
\int_{t=0}^{\infty} \int\left[\left(i \frac{\partial \psi}{\partial t}-d \psi\right)^{*} t \psi-(t \psi)^{*}\left(i \frac{\partial \psi}{\partial t}-d \psi\right)\right] \mathrm{d} V \mathrm{~d} t=i
$$

The uncertainty relation for time can be written in form analogous to Eq. (28)

$$
\int_{0}^{\infty} \int t^{2}|\psi|^{2} \mathrm{~d} V \mathrm{~d} t \int_{0}^{\infty} \int|i \partial \psi / \partial t-d \psi|^{2} \mathrm{~d} V \mathrm{~d} t \geq \frac{1}{4}
$$

Minimum of the left side is obtained for

$$
d=\frac{1}{2}\left[\int_{0}^{\infty} \int \psi^{*} i(\partial \psi / \partial t) \mathrm{d} V \mathrm{~d} t+c . c .\right] .
$$

Equation (36) is valid also if $d$ is replaced by a real function $f_{0}(\mathbf{r}, t)$. 
To illustrate meaning of Eq. (36) we assume decaying probability amplitude with the life time $\tau>0$

$$
\psi(\mathbf{r}, t)=\frac{1}{\sqrt{\tau}} \mathrm{e}^{-i \omega t-t /(2 \tau)} \psi(\mathbf{r}),
$$

where the space part of the probability amplitude is normalized by the usual condition $\int|\psi(\mathbf{r})|^{2} \mathrm{~d} V=1$. In this case, we get from Eqs. (36)-(37)

$$
\int_{0}^{\infty} \int t^{2}|\psi|^{2} \mathrm{~d} V \mathrm{~d} t=2 \tau^{2}
$$

$d=\omega$ and

$$
\int_{0}^{\infty} \int|i \partial \psi / \partial t-d \psi|^{2} \mathrm{~d} V \mathrm{~d} t=\frac{1}{4 \tau^{2}}
$$

Therefore, uncertainty relation (36) gives the relation between the mean square time $\left\langle t^{2}\right\rangle=2 \tau^{2}$ and the square of the imaginary part of the complex frequency $\omega-i /(2 \tau)$ and has meaning of the time-energy uncertainty relation.

In agreement with our understanding of direction of time, we assume that direct physical meaning have only the probability amplitudes corresponding to the non-negative values of the time component of the probability density current integrated over the whole space

$$
\int j_{t} \mathrm{~d} V=-\int \rho \frac{\partial s}{\partial t} \mathrm{~d} V \geq 0
$$

If this quantity is negative, its sign can be reversed by the transformation $\psi \rightarrow \psi^{*}$ changing the sign of the phase $s$ and the probability density currents $j_{k}$ and $j_{t}$. Performing this transformation we get from Eq. (35) for $d=f_{0}$

$$
\int_{t=0}^{\infty} \int\left[\left(i \frac{\partial \psi}{\partial t}+f_{0} \psi\right)^{*} t \psi-(t \psi)^{*}\left(i \frac{\partial \psi}{\partial t}+f_{0} \psi\right)\right] \mathrm{d} V \mathrm{~d} t=i
$$

and see that this transformation changes the sign of $f_{0}$.

Similar discussion can be done also for the space coordinates. As a result, the transformation $\psi \rightarrow \psi^{*}$ leads to change of sign of the functions $f_{0}$ and $f_{k}, k=1,2,3$ that can be respected by putting $f_{0}=e U$ and $f_{k}=e A_{k}$, where $U$ and $A_{k}$ are the scalar and vector electromagnetic potentials. Therefore, the probability amplitudes $\psi$ and $\psi^{*}$ describe particles that differ by the sign of their charge and existence of particles and antiparticles agrees with general structure of the probability theory and unidirectional character of time.

Except for $\hbar$, we obtained also the rules $i \hbar \partial / \partial t \rightarrow i \hbar \partial / \partial t-e U$ and $-i \hbar \nabla \rightarrow-i \hbar \nabla-e \mathbf{A}$ for including the electromagnetic potentials into quantum theory. These potentials representing different physical scenarios do not appear among the variables of the probability amplitude and describe non-quantized classical fields.

Now, we perform transition to standard quantum mechanics. In this limit case the integration over time need not be performed and the probability density can be normalized over the space only $\int|\psi|^{2} \mathrm{~d} V=1$. At the same time, the uncertainty relation (36) losts its original meaning and time becomes a parameter rather than a dynamical variable. It is the first reason for a different role of time and space coordinates in quantum mechanics. The second reason is that the operator $i \partial / \partial t$ appears in equations of motion like the Schrödinger equation and does not represent an independent physical quantity.

It is worth noting that to obtain results of sections IIVI no evolution equation has been needed. Therefore, this part of the mathematical formalism of quantum mechanics follows directly from the probabilistic description of results of measurements. It is also interesting that the Planck constant $\hbar$ does not appear in our discussion and can be included by multiplying Eqs. (21) and (34) by $\hbar$. Therefore, the Planck constant determines the units used in measurements and scales at which the probabilistic character of measurements is important.

\section{EQUATIONS OF MOTION}

To find equations of motion we require relativistic invariance of the theory. Our approach is similar to that used by Frieden who derives basic equations of physics from the principle of extreme physical information [8].

First we note that all quantities discussed above depend on $\psi$ or its first derivatives with respect to time and space coordinates. Returning back to our scheme used in section $\nabla \mathbb{1}$ we can create real relativistic invariant from the first derivatives of $\psi$ appearing in the uncertainty relations (28) and (36) for $a=b=d=0$

$$
\int_{0}^{\infty} \int\left(\frac{1}{c^{2}}\left|\frac{\partial \psi}{\partial t}\right|^{2}-\sum_{k=1}^{3}\left|\frac{\partial \psi}{\partial x^{k}}\right|^{2}\right) \mathrm{d} V \mathrm{~d} t=\text { const }
$$


where $c$ is the speed of light.

Integral $\int_{0}^{\infty} \int|\partial \psi / \partial t|^{2} \mathrm{~d} V \mathrm{~d} t$ has meaning of the time Fisher information and is non-negative. Similar conclusion applies also for $\int_{0}^{\infty} \int\left|\partial \psi / \partial x^{k}\right|^{2} \mathrm{~d} V \mathrm{~d} t, k=1,2,3$. However, since Eq. (43) must be valid in all cases including the case $\partial \psi / \partial x^{k}=0$ (in the language of quantum mechanics, it corresponds to zero momentum and zero kinetic energy) we can conclude that const $\geq 0$.

In this equation, we can perform integration by parts with respect to all variables. For example, we get for time

$$
\int_{0}^{\infty} \int \frac{\partial \psi^{*}}{\partial t} \frac{\partial \psi}{\partial t} \mathrm{~d} V \mathrm{~d} t=\frac{1}{2}\left[\int\left(\psi^{*} \frac{\partial \psi}{\partial t}+\text { c.c. }\right) \mathrm{d} V\right]_{0}^{\infty}-\frac{1}{2} \int_{0}^{\infty} \int\left(\psi^{*} \frac{\partial^{2} \psi}{\partial t^{2}}+\text { c.c. }\right) \mathrm{d} V \mathrm{~d} t .
$$

However, the first integral on the right side can be expressed as $\partial\left(\int|\psi|^{2} \mathrm{~d} V\right) / \partial t$ and disappears in the limit of standard quantum mechanics when $\int|\psi|^{2} \mathrm{~d} V=1$. Analogous result can be obtained also for the variables $x^{k}$ assuming that $\partial|\psi|^{2} / \partial x^{k}$ for $x^{k} \rightarrow-\infty$ and $x^{k} \rightarrow \infty$ equal. In standard quantum mechanics, this condition is obeyed for a free particle as well as for the bound states.

Now, we perform transition to standard quantum mechanics with the wave function normalized in the usual way and get

$$
\frac{1}{2} \int\left[\psi^{*}\left(\Delta-\frac{1}{c^{2}} \frac{\partial^{2}}{\partial t^{2}}-\text { const }\right) \psi+\text { c.c. }\right] \mathrm{d} V=0 .
$$

From here, we get equation of motion in the form

$$
\left(\Delta-\frac{1}{c^{2}} \frac{\partial^{2}}{\partial t^{2}}-\text { const }\right) \psi=0
$$

Since const $\geq 0$, we can put const $=m_{0}^{2} c^{2} / \hbar^{2}$, where $m_{0}$ is another constant, known as the rest mass of the particle. Therefore, requirement of the relativistic invariance applied to quantities appearing in the probabilistic formulation leads to the Klein-Gordon equation for a free particle.

The non-relativistic time Schrödinger equation can be obtained from the Klein-Gordon equation by using the transformation

$$
\psi=\mathrm{e}^{m_{0} c^{2} t /(i \hbar)} \varphi
$$

where $\varphi$ is the probability amplitude appearing in the Schrödinger equation. This transition is known and will not be discussed here [11].

The Dirac equation can be derived by replacing the probability amplitude $\psi$ in Eq. (43) by a column vector with four components

$$
\int_{0}^{\infty} \int\left(\frac{1}{c^{2}} \frac{\partial \psi^{+}}{\partial t} \frac{\partial \psi}{\partial t}-\sum_{k=1}^{3} \frac{\partial \psi^{+}}{\partial x^{k}} \frac{\partial \psi}{\partial x^{k}}\right) \mathrm{d} V \mathrm{~d} t=\text { const }
$$

where the cross denotes the hermitian conjugate. Inserting the $\gamma^{\mu}$ matrices [1] into this equation, putting const $=$ $m_{0}^{2} c^{2} / \hbar^{2}$ and using Eq. (31) we get

$$
\int_{0}^{\infty} \int\left[\frac{1}{c^{2}}\left(\gamma^{0} \frac{\partial \psi}{\partial t}\right)^{+}\left(\gamma^{0} \frac{\partial \psi}{\partial t}\right)-\sum_{k=1}^{3}\left(\gamma^{k} \frac{\partial \psi}{\partial x^{k}}\right)^{+}\left(\gamma^{k} \frac{\partial \psi}{\partial x^{k}}\right)-\frac{m_{0}^{2} c^{2}}{\hbar^{2}} \psi^{+} \psi\right] \mathrm{d} V \mathrm{~d} t=0 .
$$

Then, using properties of the $\gamma^{\mu}$ matrices and assuming that the integration by parts can be used analogously as in case of Eq. (44) the last equation leads in the limit of standard quantum mechanics to (see also [7, 8] )

$$
\int\left(\frac{\gamma^{0}}{c} \frac{\partial \psi}{\partial t}-\sum_{k=1}^{3} \gamma^{k} \frac{\partial \psi}{\partial x^{k}}-\frac{i m_{0} c}{\hbar} \psi\right)^{+}\left(\frac{\gamma^{0}}{c} \frac{\partial \psi}{\partial t}+\sum_{k=1}^{3} \gamma^{k} \frac{\partial \psi}{\partial x^{k}}+\frac{i m_{0} c}{\hbar} \psi\right) \mathrm{d} V=0
$$

The operator in the first parentheses is the hermitian conjugate of that in the second ones. Assuming that the expression in the second parentheses equals zero we obtain the Dirac equation for a free particle

$$
\frac{\gamma^{0}}{c} \frac{\partial \psi}{\partial t}+\sum_{k=1}^{3} \gamma^{k} \frac{\partial \psi}{\partial x^{k}}+\frac{i m_{0} c}{\hbar} \psi=0
$$

We see that requirement of the relativistic invariance of the probabilistic description yields all the basic equations of motion of quantum mechanics. The scalar and vector potentials can be included by means of the rules $i \hbar \partial / \partial t \rightarrow$ $i \hbar \partial / \partial t-e U$ and $-i \hbar \nabla \rightarrow-i \hbar \nabla-e \mathbf{A}$ discussed above. 


\section{CLASSICAL MECHANICS}

To derive the Hamilton-Jacobi equation for a free particle we proceed as follows. The probability amplitude is assumed in the form

$$
\psi=\mathrm{e}^{i s / \hbar}=\mathrm{e}^{i s_{1} / \hbar} \mathrm{e}^{-s_{2} / \hbar}
$$

where $s_{1}$ and $s_{2}$ are the real and imaginary parts of $s$, respectively. In the limit of standard quantum mechanics mentioned above, Eq. (43) with const $=m_{0}^{2} c^{2} / \hbar^{2}$ can be replaced by the equation

$$
\frac{1}{c^{2}} \int\left|\frac{\partial s}{\partial t}\right|^{2}|\psi|^{2} \mathrm{~d} V=\int|\nabla s|^{2}|\psi|^{2} \mathrm{~d} V+m_{0}^{2} c^{2}
$$

Now we assume that the probability density

$$
\rho=|\psi|^{2}=\mathrm{e}^{-2 s_{2} / \hbar}
$$

has very small values everywhere except for the vicinity of the point $\langle\mathbf{r}\rangle$, where it achieves its maximum and the first derivatives of $s_{2}$ at this point equal zero

$$
\left.\frac{\partial s_{2}}{\partial x^{k}}\right|_{\mathbf{r}=\langle\mathbf{r}\rangle}=0, \quad k=1,2,3 .
$$

In such a case, the probability density can be replaced by the $\delta$-function

$$
|\psi|^{2}=\delta(\mathbf{r}-\langle\mathbf{r}\rangle)
$$

and probabilistic character of the theory disappears. Equations (53)-(56) then lead to the relativistic equation

$$
\frac{1}{c^{2}}\left(\frac{\partial s_{1}(\langle\mathbf{r}\rangle,\langle t\rangle)}{\partial t}\right)^{2}=\left[\nabla s_{1}(\langle\mathbf{r}\rangle, t)\right]^{2}+m_{0}^{2} c^{2}
$$

We note that Eq. (56) corresponds to the limit $\hbar \rightarrow 0$ in Eq. (54). Therefore, $s_{1}$ in Eq. (57) is in fact the first term of the expansion of $s_{1}$ into the power series in $\hbar$

$$
s_{1}=\left.s_{1}\right|_{\hbar=0}
$$

Further, we replace the mean coordinates $\langle\mathbf{r}\rangle$ by $\mathbf{r}$ as it is usual in classical mechanics and introduce the classical non-relativistic action $S(\mathbf{r}, t)$

$$
s_{1}=S-m_{0} c^{2} t
$$

Equation (57) then leads to

$$
\frac{1}{c^{2}}\left(\frac{\partial S}{\partial t}-m_{0} c^{2}\right)^{2}=(\nabla S)^{2}+m_{0}^{2} c^{2}
$$

In the non-relativistic limit $|\partial S / \partial t| \ll m_{0} c^{2}$ the last equation yields the Hamilton-Jacobi equation for a free particle

$$
\frac{\partial S}{\partial t}+\frac{(\nabla S)^{2}}{2 m_{0}}=0
$$

Thus, the Hamilton-Jacobi equation can be obtained from the probabilistic description of measurements in the limit of $\delta$-like probability densities and non-relativistic approximation. The scalar and vector potentials $U$ and $\mathbf{A}$ can be included by means of the rules $\partial S / \partial t \rightarrow \partial S / \partial t+e U$ and $\nabla S \rightarrow \nabla S-e \mathbf{A}$ following from the rules $i \hbar \partial / \partial t \rightarrow i \hbar \partial / \partial t-e U$ and $-i \hbar \nabla \rightarrow-i \hbar \nabla-e \mathbf{A}$ discussed above. 


\section{MANY PARTICLE SYSTEMS}

In general, many particle systems have to be described by the quantum field theory. However, if we limit ourselves to quantum mechanics, we can proceed as follows.

The starting point of discussion of the $N$ particle system is definition analogous to Eq. (1)

$$
\left\langle\mathbf{r}_{j}\right\rangle=\int \mathbf{r}_{j} \rho\left(\mathbf{r}_{1}, \ldots, \mathbf{r}_{N}, t\right) \mathrm{d} V_{1} \ldots \mathrm{d} V_{N}, j=1, \ldots, N,
$$

where $\rho$ is the many particle probability density and $\mathbf{r}_{j}$ are the coordinates of the $j$-th particle. Then, discussion can be performed analogously to that given above and the probability amplitude, uncertainty and commutation relations, momentum operators and density currents for all particles can be introduced. The scalar and vector potentials $U\left(\mathbf{r}_{1}, \ldots, \mathbf{r}_{N}, t\right)$ and $\mathbf{A}\left(\mathbf{r}_{1}, \ldots, \mathbf{r}_{N}, t\right)$ and antiparticles can be also discussed.

Equations of motion for $N$ free particles can be found from generalization of the relativistic invariant (43)

$$
\int_{0}^{\infty} \int\left(\frac{1}{c^{2}}\left|\frac{\partial \psi}{\partial t}\right|^{2}-\sum_{j=1}^{N}\left|\nabla_{j} \psi\right|^{2}\right) \mathrm{d} V_{1} \ldots \mathrm{d} V_{N} \mathrm{~d} t=\sum_{j=1}^{N} \frac{m_{j}^{2} c^{2}}{\hbar^{2}},
$$

where $\psi\left(\mathbf{r}_{1}, \ldots, \mathbf{r}_{N}, t\right)$ is the $N$ particle probability amplitude and $m_{j}$ denotes the rest mass of the particle.

Using similar approach as above, we can then obtain the $N$ particle Schrödinger equation

$$
-\sum_{j=1}^{N} \frac{\hbar^{2}}{2 m_{j}} \Delta_{j} \psi=i \hbar \frac{\partial \psi}{\partial t}
$$

and the Hamilton-Jacobi equation

$$
\frac{\partial S}{\partial t}+\sum_{j=1}^{N} \frac{\left(\nabla_{j} S\right)^{2}}{2 m_{j}}=0
$$

For a system of identical particles, the probability density $\rho$ must be symmetric with respect to the exchange of any two particles $i$ and $j$. Hence, the probability amplitude $\psi$ must be symmetric or antisymmetric with respect to such exchanges.

Non-locality of quantum mechanics is related to the many particle character of the probability density $\rho$ and the corresponding probability amplitude $\psi$.

It is seen that probabilistic description of measurements and its relativistic invariance yields also the basic mathematical structure of the many particle quantum mechanics.

\section{CONCLUSIONS}

In this paper, we have shown that the basic mathematical structure of quantum mechanics can be derived from the probabilistic description of the results of measurement of the space coordinates and time. Equations of motion of quantum mechanics have been obtained from requirement of the relativistic invariance of the theory. As a limit case, this approach yields also the Hamilton-Jacobi equation of classical mechanics.

Since our approach makes possible to obtain the most significant parts of the mathematical formalism of quantum mechanics from the probabilistic description of results of measurements, we believe that it is natural and physically satisfactory starting point to understanding this field. It contributes also to understanding quantum theory as correctly formulated probabilistic description of measurements that can describe physical phenomena at different levels of accuracy from the most simple models to very complex ones.

\section{Acknowledgments}

This work was supported by GA CR (grant No. 202/03/0799) and MS (grant No. 190-01/206053) of the Czech Republic.

[1] A. Zeilinger, Rev. Mod. Phys. 71 (1999) S288. 
[2] M. Arndt, O. Nairz, A. Zeilinger, in Quantum [Un]speakables. From Bell to Quantum Information, edited by R. A. Bertlmann, A. Zeilinger (Springer, Berlin, 2002).

[3] M. O. Scully, B. G. Englert, H. Walther, Nature 351 (1991) 111.

[4] F. Laloë, Am. J. Phys. 69 (2001) 655.

[5] Ch. A. Fuchs, A. Peres, Physics Today (March 2000) 70.

[6] R. A. Fisher, Proc. Cambr. Phil. Soc. 22 (1925) 700.

[7] B. Roy Frieden, B. H. Soffer, Phys. Rev. E 52 (1995) 2274.

[8] B. Roy Frieden, Physics from Fisher information (Cambridge University Press, Cambridge, 1998).

[9] T. Cover, J. Thomas, Elements of Information Theory (Wiley, New York, 1991).

[10] B. Roy Frieden, Phys. Lett. A 169 (1992) 123.

[11] A. S. Davydov, Quantum Mechanics (Pergamon Press, New York, 1976). 